\title{
In Vitro Bioconversion of Polyphenols from Black Tea and Red Wine/Grape Juice by Human Intestinal Microbiota Displays Strong Interindividual Variability
}

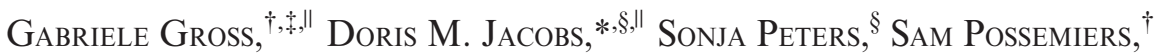 \\ John van Duynhoven, ${ }^{\S}$ Elaine E. Vaughan, ${ }^{*}$ and Tom van de Wiele ${ }^{\dagger}$ \\ ${ }^{\dagger}$ Laboratory of Microbial Ecology and Technology (LabMET), Faculty of Bioscience Engineering, \\ Ghent University, Coupure Links 653, B-9000 Gent, Belgium, "Biosciences, Unilever R\&D, \\ Olivier van Noortlaan 120, 3130 AC Vlaardingen, The Netherlands, and ${ }^{\S}$ Advanced Measurement \\ and Data Modelling, Unilever R\&D, Olivier van Noortlaan 120, 3130 AC Vlaardingen, \\ The Netherlands. "These authors equally contributed to the manuscript.
}

\begin{abstract}
Dietary polyphenols in tea and wine have been associated with beneficial health effects. After ingestion, most polyphenols are metabolized by the colonic microbiota. The current study aimed at exploring the interindividual variation of gut microbial polyphenol bioconversion from 10 healthy human subjects. In vitro fecal batch fermentations simulating conditions in the distal colon were performed using polyphenols from black tea and a mixture of red wine and grape juice. Microbial bioconversion was monitored by NMR- and GC-MS-based profiling of diverse metabolites and phenolics. The complex polyphenol mixtures were degraded to a limited number of key metabolites. Each subject displayed a specific metabolite profile differing in composition and time courses as well as levels of these metabolites. Moreover, clear differences depending on the polyphenol sources were observed. In conclusion, varying metabolite pathways among individuals result in different metabolome profiles and therefore related health effects are hypothesized to differ between subjects.
\end{abstract}

KEYWORDS: Fecal batch fermentations; gut microbiota; interindividual variation; metabolomics; tea/wine polyphenols

\section{INTRODUCTION}

Epidemiologic data as well as experimental studies suggest health benefits for dietary polyphenols that are abundantly present in foods such as tea and wine (1). Polyphenols that are initially absorbed in the ileum can subsequently be excreted as conjugates in the bile and pass through the small intestine before they arrive in the colon. However, a major portion of nonabsorbed polyphenols reaches the colon directly after gastrointestinal passage (2), where they can be deconjugated and fermented by the gut microbiota to a wide range of low-molecular-weight metabolites, which may be absorbed by the host (3).

The microbial community in the human colon is highly diverse: it comprises $10^{12}$ bacteria/g of colonic content belonging to hundreds of different species and exerts a profound impact on human health $(4,5)$. The composition of the gut microbiota varies substantially among individuals (6). Consequently, each intestinal microbial community can be expected to display its own characteristic metabolic profile. It has been reported that particular beneficial effects of polyphenol-rich foods are dependent on biotransformation by intestinal bacteria to bioactive metabolites (7). For some polyphenolic compounds, bioconversion has been shown to vary between individual persons due to differences

*To whom correspondence should be addressed. Phone: +31104605987. Fax: +31 10460 5310. E-mail: doris.jacobs@unilever.com. in the metabolic potential of their endogenous microbiota (8). However, the underlying mechanisms and bacterial species involved in polyphenol breakdown have not yet been identified sufficiently (for review see ref 3 ), and the bacterial metabolism of complex mixtures of polyphenolic substances as present in the human diet has been studied far less extensively than that of single compounds. For instance, black tea and red wine/grape polyphenol extracts are complex mixtures with only partially known composition. Basically, red wine polyphenols mainly contain proanthocyanidins, anthocyanins, catechins, flavonols, and some phenolic acids, whereas black tea polyphenols are primarily composed of thearubigins, theaflavins, catechins, flavonols, and phenolic acids $(9,10)$.

A detailed overview of bacterial polyphenol metabolites gained with metabolomics technologies can increase our understanding of the bioconversion of dietary compounds by the intestinal microbiota and would contribute to unravelling the mechanisms of beneficial health effects associated with dietary polyphenols. To this end, in vitro batch fermentations were performed in which fecal samples from 10 human volunteers were incubated with polyphenol extracts from black tea and a mixture of red wine and grape juice (henceforth called wine/grape extract), respectively. Culture models of human colonic microbiota that simulate microbial processes in the large intestine have proven to be useful tools to investigate the extensive microbial metabolism of natural 
polyphenols (11). In contrast to more sophisticated, but timeconsuming in vitro gut models, batch fermentations provide a feasible possibility to assess multiple experimental conditions by using fecal samples from several subjects (12). Therefore, they allow comparison of microbial fermentation processes of different human microbial communities under simulated conditions as present in the distal colon by using metabolite profiling methods (13). The wide range of bacterial breakdown products was monitored over time using two metabolite profiling techniques. Nuclear magnetic resonance (NMR)-based metabolite profiling, a fast and broad, but rather insensitive profiling technique, was employed to detect changes of the major degradation products, including some phenolic metabolites and short chain fatty acids (SCFAs). In addition, a more sensitive technique, gas chromatography-mass spectrometry (GC-MS)-based profiling of phenolics, was used to focus on this specific compound class. Both techniques have been recently established for human intestinal samples in our laboratory $(14,15)$. With these combined methods, key metabolites were identified and the interindividual variability between microbial metabolic pathways of polyphenols from tea and wine/grape was characterized.

\section{MATERIALS AND METHODS}

Batch Culture Fermentations. In vitro fermentation experiments were performed under conditions as used in previous studies $(8,16)$. Fresh fecal samples were obtained from 10 healthy free-living volunteers with no dietary restrictions (six female, four male; aged 25-34 years; Caucasian, no recent history of gastrointestinal disease). The samples were transported under aerobic conditions and further processed to anaerobic conditions within maximum $1 \mathrm{~h}$, in most cases within a few minutes. For this, $20 \mathrm{~g}$ of the fecal matter was homogenized with a stomacher in $100 \mathrm{~mL}$ of phosphate buffer containing sodium thioglycolate as reducing agent. The particulate material was removed by brief centrifugation. The experiments were performed in serum bottles containing $44.5 \mathrm{~mL}$ of sterile basal medium (17) with doubled amounts of $\mathrm{NaHCO}_{3}, \mathrm{~K}_{2} \mathrm{HPO}_{4}$, and $\mathrm{KH}_{2} \mathrm{PO}_{4}$ to enhance buffering capacity. Prior to addition of $5 \mathrm{~mL}$ of the fecal slurries (or phosphate buffer as a control), the bottles were sealed with rubber tops, and anaerobiosis was obtained by flushing with $\mathrm{N}_{2}$ during 20 cycles of 2 min at 700 mbar overpressure and 900 mbar under pressure. After addition of $0.5 \mathrm{~mL}$ of dissolved polyphenol extracts or distilled water as control (referred to as "blank experiment"), the suspensions were mixed by shaking and incubated at $37^{\circ} \mathrm{C}$ for $72 \mathrm{~h}$, with sampling at baseline $(0 \mathrm{~h})$ and after 2, 4, 8, 24, 32, 48, and $72 \mathrm{~h}$. Samples were collected using syringes and stored at $-80{ }^{\circ} \mathrm{C}$ within $5 \mathrm{~min}$ in closed tubes. Samples were kept frozen at all times prior to the use of $0.5 \mathrm{~mL}$ aliquots for NMR and GC-MS analysis. The $\mathrm{pH}$ of the samples was measured at time points $0,8,24,32,48$, and $72 \mathrm{~h}$.

Two different polyphenol sources, a mixture of wine and grape juice extract and a black tea extract, were used. The wine/grape extract was composed of two parts of red wine extract Provinols (Seppic, France) and one part of MegaNatural Rubired grape juice extract (Polyphenolics, USA). The tea extract was prepared by spray-drying from Lipton Yellow label black tea (Unilever, The Netherlands). The wine/grape extract and tea extract were taken from single batches and contained $57 \%$ and $32 \%$ total polyphenols, respectively. Stock solutions were prepared by dissolving $1762.5 \mathrm{mg}$ wine/grape extract powder and $3125.0 \mathrm{mg}$ tea extract powder in $10 \mathrm{~mL}$ of distilled water, respectively, and additionally diluted 1:2 with distilled water to obtain lower concentrations. These solutions were stored at $4{ }^{\circ} \mathrm{C}$ until further use. In the experiments, $0.5 \mathrm{~mL}$ of the stock solution were added to obtain a total volume of $50 \mathrm{~mL}$ in the penicillin flasks, yielding final polyphenol concentrations of 500 and 1000 $\mathrm{mg} / \mathrm{L}$, respectively. Comparable concentrations have been used in previous experiments $(17,18)$, aiming at simulation of physiological conditions because the total dietary polyphenol intake for humans has been estimated to be approximately $1 \mathrm{~g}$ per day (19).

NMR Analysis. The thawed samples were centrifuged (5 min, $22000 g$, $8{ }^{\circ} \mathrm{C}$ ). NMR samples were prepared by adding $50 \mu \mathrm{L}$ of deuterium oxide $\left(\mathrm{D}_{2} \mathrm{O}\right)$ containing $10 \mathrm{mM}$ deuterated sodium trimethylsilyl propionate
(d-TSP) to $450 \mu \mathrm{L}$ supernatant. $\mathrm{D}_{2} \mathrm{O}$ and d-TSP serve as field frequency lock and chemical shift reference, respectively.

One-dimensional (1D) high-resolution ${ }^{1} \mathrm{H}$ NMR spectra were acquired on a Bruker Avance 600 NMR spectrometer operating at a proton NMR frequency of $600.13 \mathrm{MHz}$ and at a temperature of $300 \mathrm{~K}$. A $5 \mathrm{~mm}$ TXI probe and a sample changer for sample delivery were used. A noesypresat pulse sequence with 32k data points and 64 scans over $8993 \mathrm{~Hz}$ was used. Water suppression was achieved during the relaxation delay ( $3 \mathrm{~s}$ ) and the mixing time $(150 \mathrm{~ms})$. The spectra were manually corrected for phase and baseline distortions using Topspin 1.3 software (Bruker Analytik, Rheinstetten, Germany). An exponential window function with a line-broadening factor of $0.3 \mathrm{~Hz}$ was applied to the free induction decay (FID) prior to Fourier transformation. The spectra were referenced to TSP $\left(\delta \mathrm{CH}_{3}\right.$ : $0 \mathrm{ppm})$. Metabolites were identified using an in-house database and a database (bbiorefcode-2-0-0 implemented in Amix 3.7.3., Bruker Biospin $\mathrm{GmbH}$, Germany) including reference spectra of metabolites at different $\mathrm{pH}$ values. Metabolites were quantified using Chenomx NMR Suite 5.1. (Chenomx Inc., Edmonton, Canada). Assignment of signals was mainly performed as described previously (14).

GC-MS Analysis. The production of phenolic acids was profiled using GC-MS as described earlier (15). In particular, $0.4 \mathrm{~mL}$ of the thawed samples were first centrifuged at $22000 \mathrm{~g}$ for $5 \mathrm{~min}$ at $8^{\circ} \mathrm{C}$. T-cinnamic acid$d_{6}(100 \mu \mathrm{g} / \mathrm{mL}$ in $1: 1$ methanol/water $(\mathrm{v} / \mathrm{v}))$ was added as internal standard. The solutions were acidified with $2 \mathrm{M} \mathrm{HCl}$ and stored at $4{ }^{\circ} \mathrm{C}$ for $10 \mathrm{~min}$. Subsequently, $2 \mathrm{mLof}$ ethyl acetate were added and the tubes were vortex-mixed for $30 \mathrm{~s}$, followed by centrifugation at $3000 \mathrm{~g}$ for $10 \mathrm{~min}$. The supernatants were transferred, and the extraction was repeated twice. Extracts were combined and dried under a stream of nitrogen using a heating block at $40{ }^{\circ} \mathrm{C}$. Further drying was performed by subsequent addition and evaporation of $1 \mathrm{~mL}$ of dichloromethane. The dry samples were derivatized with $100 \mu \mathrm{L}$ of $N, O$-bis(trimethylsilyl)trifluoroacetamide (BSTFA) $+10 \%$ trimethylchlorosilane (TMCS) for 30 min at $90^{\circ} \mathrm{C}$. After derivatization, $400 \mu \mathrm{L}$ of $n$-hexane were added to the samples and after vortex-mixing, $200 \mu \mathrm{L}$ were transferred to GC vials for analysis by GC-MS.

The calibration standards were prepared from a phenolic acid stock solution containing 33 phenolic acids (Table S1, Supporting Information) at a concentration of $100 \mu \mathrm{g} / \mathrm{mL}$. The stock solution was diluted with a $1: 1$ methanol/water $(\mathrm{v} / \mathrm{v})$ solution to the desired concentration using the following dilution factors: 10, 20, 100, 200, 500, 1000, 2000, 5000, 10000. For each calibration series, $50 \mu \mathrm{L}$ of the respective standard plus the internal standard at a concentration of $5 \mu \mathrm{g} / \mathrm{mL}$ were dried under a stream of liquid nitrogen and further dried using two times $1 \mathrm{~mL}$ of dichloromethane. Further processing was performed in the same way as the experimental samples.

Derivatized samples were analyzed with a time-of-flight mass analyzer (Waters Micro Mass GCT accurate mass spectrometer) interfaced with an Agilent 6890 gas chromatograph and with a Hewlett-Packard 7683 autosampler. The injection port was kept at $55^{\circ} \mathrm{C}$ for $1 \mathrm{~min}$ and then increased to 300 at $10^{\circ} \mathrm{C} / \mathrm{s}$. The MS source and the GC-MS interface were kept at 180 and $250{ }^{\circ} \mathrm{C}$, respectively. Separations were carried out on a Varian Factor Four fused silica capillary column $(30 \mathrm{~m} \times 0.25 \mathrm{~mm}$ i.d.) coated with cross-linked 5\% phenyl methyl dimethylpolysiloxane (film thickness $0.1 \mu \mathrm{m})$. Helium was the carrier gas with a constant flow of $1 \mathrm{~mL} / \mathrm{min}$. Derivatized samples ( $1 \mu \mathrm{L}$, using a $5 \mu \mathrm{L}$ syringe $)$ were injected into the GC injection port using a split ratio of 1:10. The column temperature was kept at $45^{\circ} \mathrm{C}$ for $1 \mathrm{~min}$, then increased from 45 to $100{ }^{\circ} \mathrm{C}$ at $10{ }^{\circ} \mathrm{C} / \mathrm{min}$, then from $100^{\circ} \mathrm{C}$ to 250 at $7.5^{\circ} \mathrm{C} / \mathrm{min}$, then from 250 to $300^{\circ} \mathrm{C}$ at $20^{\circ} \mathrm{C} / \mathrm{min}$, where it was held for $6 \mathrm{~min}$. Full scanning mass spectrometry was applied for all analysis using the electron-ionization (EI) mode at $70 \mathrm{eV}$. For calibration, the phenolic acid standard mixture was analyzed with each sample set or once per week, as applicable.

Statistical Analysis. Mean values, standard deviations, and coefficient of variations $(\mathrm{CV}=\mathrm{SD} /$ mean) were calculated from the total amount of variability metabolites produced within $48 \mathrm{~h}$ (AUC in $\mathrm{mM} \cdot \mathrm{h}$ ) of fermentations with tea and wine/grape extract, respectively. To estimate the interindividual variation in the metabolite production from fermentations with tea and wine/grape extract, respectively, correlation matrices (Pearson) were calculated on AUC values and scaled to univariance and using a significance level of $p<0.01$.

Box plots including median, minimum, maximum, 25th quartile, and 75th quartile values were calculated for the concentrations of several 


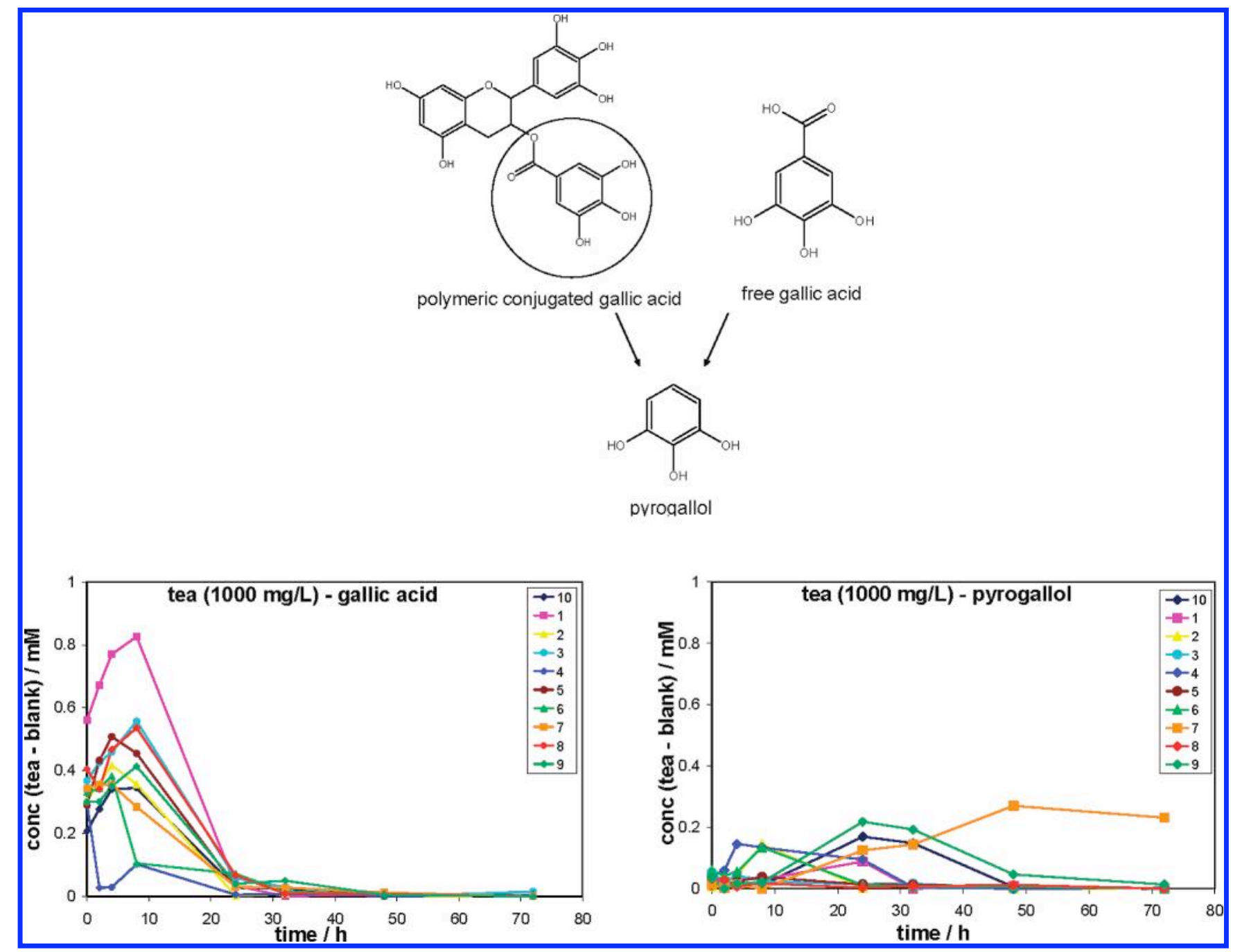

Figure 1. Conversion of free gallic acid and conjugated gallic acid in epigallocatechingallate (EGCG) and other gallo-tannins potentially present in black tea extract $(3,20)$ (left lower panel) to pyrogallol (right lower panel; both derived from ${ }^{1} \mathrm{H}$ NMR spectra). The kinetic curves from each subject are displayed by different colors. The concentration derived from the blank experiment was subtracted from the concentration derived from the experiments using the tea extract (conc (tea-blank)/mM).

SCFAs as determined from fermentation experiments using $1000 \mathrm{mg} / \mathrm{L}$ polyphenols after $72 \mathrm{~h}$. An ANOVA test was performed to assess statistically significant effects for each of the SCFAs.

\section{RESULTS}

Combined Metabolite Profiling Methods Provide Comprehensive Information on Microbial Polyphenol Bioconversion under Batch Fermentation Conditions. In vitro batch culture fermentations were performed using colonic microbiota from 10 healthy volunteers. With each fecal sample, five batch fermentations were performed with either the wine/grape or tea extract added at a concentration of 500 and $1000 \mathrm{mg} / \mathrm{L}$, respectively, or water as a negative control. $\mathrm{pH}$ measurements at regular time points revealed a maximum average decrease in $\mathrm{pH}$ among the different treatments of 0.46 units between time point $0 \mathrm{~h}$ and $72 \mathrm{~h}$, demonstrating that the $\mathrm{pH}$ remained relatively stable throughout the incubation (average of all treatments \pm standard deviation: $\mathrm{pH} 7.15 \pm 0.07$ at $0 \mathrm{~h} ; \mathrm{pH} 6.92 \pm 0.26$ at $72 \mathrm{~h}$ ).

Supernatants of all batch fermentations were analyzed using NMR-based metabolite profiling. The complex NMR profiles comprised different classes of compounds such as major phenolic acids, SCFAs, alcohols, and amines. The profiles generated from incubations with $1000 \mathrm{mg} / \mathrm{L}$ polyphenols were generally used to quantify the major phenolic metabolites due to their higher signal-to-noise-ratios. Figure S1 of Supporting Information illustrates how the bioconversion of polyphenols could be traced with this method over a period of time using signals originating from initial substances, intermediate and end products, and invariant compounds. However, the profiles obtained from the blank experiments showed that nutrients from the medium were consumed over time and converted to intermediate products, such as ethanol and lactate, and end products, mainly SCFAs, phenylacetic acid, and 3-phenylpropionic acid (data not shown). Therefore, the values obtained from the incubation with the polyphenol extracts were corrected for the corresponding blank values, resulting in metabolite changes that can be solely attributed to polyphenol bioconversion. Separate incubations of the medium with polyphenol extracts but without fecal inoculum served as additional controls and confirmed that the signals did not arise from nonmicrobial degradation processes (data not shown).

In comparison to NMR-based metabolite profiling, GC-MS is a more sensitive method. Therefore, samples originating from incubations with the lower initial ingredient concentration of 500 $\mathrm{mg} / \mathrm{mL}$ were analyzed with this method. The recorded GC-MS profiles included numerous phenolic metabolites, and changes in the levels of less abundant phenolic acids such as diverse derivatives 
Table 1. Overview of Metabolite Changes from the Fermentations with Black Tea and Red Wine/Grape Juice Extracts with Respect to Blank Experiments Derived from ${ }^{1} \mathrm{H}$ NMR (Indicated by NMR) and GC-MS Profiles ${ }^{a}$

\begin{tabular}{|c|c|c|c|c|c|c|c|c|c|c|c|c|c|c|c|c|c|c|}
\hline & \multicolumn{9}{|c|}{ Tea versus blank } & \multicolumn{9}{|c|}{ Wine versus blank } \\
\hline & S1 & S3 & S4 & S5 & S6 & S7 & S8 & S9 & S10 & S1 & S3 & S4 & S5 & S6 & S7 & S8 & S9 & S10 \\
\hline \multicolumn{19}{|l|}{ 3-phenylpropionate (NMR) } \\
\hline \multicolumn{19}{|l|}{ 3-(3-OH-phenyl)propionate (NMR) } \\
\hline \multicolumn{19}{|l|}{ 3-(4-OH-phenyl)propionate (NMR) } \\
\hline \multicolumn{19}{|l|}{ 3-(3,4-diOH-phenyl)propionate } \\
\hline \multicolumn{19}{|l|}{ phenylacetate (NMR) } \\
\hline \multicolumn{19}{|l|}{$\alpha-O H p h e n y l a c e t i c$ acid (mandelic) } \\
\hline \multicolumn{19}{|l|}{ 2/4-OH-phenylacetate } \\
\hline \multicolumn{19}{|l|}{ 3-OH-phenylacetate } \\
\hline \multicolumn{19}{|l|}{ 3,4-diOH-phenylacetate } \\
\hline \multicolumn{19}{|l|}{ 3-OH-benzoic acid } \\
\hline \multicolumn{19}{|l|}{ 4-OH-benzoic acid } \\
\hline \multicolumn{19}{|l|}{ 2,3-diOH-benzoic acid } \\
\hline \multicolumn{19}{|l|}{ 2,6-diOH-benzoic acid } \\
\hline \multicolumn{19}{|l|}{ 3,4-diOH-benzoic acid } \\
\hline \multicolumn{19}{|l|}{ 3,5-diOH-benzoic acid } \\
\hline \multicolumn{19}{|l|}{ 4-OH-3-MeO-benzoic acid (vanillic) } \\
\hline \multirow{2}{*}{\multicolumn{19}{|c|}{$\begin{array}{l}\text { 3,4,5-triOH-benzoic acid (gallic)* } \\
\text { 3,4,5-triOH-benzoic acid (gallic) (NMR) }\end{array}$}} \\
\hline & & & & & & & & & & & & & & & & & & \\
\hline \multicolumn{19}{|l|}{ 1,2,3-triOH-benzene (pyrogallol) (NMR) } \\
\hline \multirow{2}{*}{\multicolumn{19}{|c|}{$\begin{array}{l}\text { 1,3,5-triOH-benzene (phloroglucinol) } \\
\text { 1,3,5-triOH-benzene (ph/oroglucinol) (NMR) }\end{array}$}} \\
\hline & & & & & & & & & & & & & & & & & & \\
\hline 3-OH-cinnamic acid (m-coumaric) & & & & & & & & & & & & & & & & & & \\
\hline 4-OH-cinnamic acid (p-coumaric)* & & & & & & & & & & & & & & & & & & \\
\hline 3,4-diOH-cinnamic acid (caffeic)* & & & & & & & & & & & & & & & & & & \\
\hline
\end{tabular}

${ }^{a}$ Color coding indicates time point of maximum reduced or increased value occurrence (reduced: dark-blue squares: $72 \mathrm{~h}$; light-blue squares: $24 \mathrm{~h}$; increased: light-yellow
squares: $0 / 2 \mathrm{~h}$; medium-yellow squares: $4 \mathrm{~h}$; dark-yellow squares: $8 \mathrm{~h}$; light-orange squares: $24 \mathrm{~h}$; dark-orange squares: $32 \mathrm{~h}$; red squares: $48 / 72 \mathrm{~h}$; $\mathrm{n} / \mathrm{a}$ : not applicable; *: present in initial extract).

of hydroxyphenylacetic acids and hydroxybenzoic acids were detected.

The two measurement approaches NMR and GC-MS complemented each other. Low abundant phenolic acids were only detectable by GC-MS, and highly abundant phenolic acids that were within the saturation limit of GC-MS were determined by NMR spectroscopy.

The Concentration of the Polyphenol Source Has a Limited Impact on the Metabolite Profile. The metabolite profiles from incubations with 500 and $1000 \mathrm{mg} / \mathrm{L}$ polyphenol extract were comparable with respect to the metabolites produced and their time courses. For example, similar time courses were obtained for the production of 3-(4-hydroxyphenyl)propionic acid after incubations with 500 and $1000 \mathrm{mg} / \mathrm{L}$ of the tea and wine/grape extract, respectively (Figure S2, Supporting Information). Slight differences in the production of 3-(4-hydroxyphenyl)propionic acid can be attributed to the different polyphenol concentrations used. For example, whereas 3-(4-hydroxyphenyl)propionic acid was found to be only temporarily produced by the microbiota of subject 9 when $500 \mathrm{mg} / \mathrm{L}$ of the tea extract was incubated, it was still detectable after $72 \mathrm{~h}$ of fermentation with $1000 \mathrm{mg} / \mathrm{L}$ of the same extract. This result demonstrates that the initial concentrations only had a minor impact on the metabolite profiles and that the incubations were generally reproducible. Only the production of pyrogallol from gallic acid present in free form or in larger polyphenols such as epigallocatechingallate in the tea extract (20) strongly depended on the initial concentration and was only observed in incubations with $1000 \mathrm{mg} / \mathrm{L}$ black tea extract. Pyrogallol was not quantitatively converted from gallic acid and further degraded by the microbiota of most subjects except for subject 7 , for whom it was still detectable after $72 \mathrm{~h}$ (Figure 1).

Polyphenol Bioconversion by Human Gut Microbiota Is Characterized by Strong Interindividual Variations. Table 1 and Figure S3 of Supporting Information provide an overview of the diversity of metabolite changes observed by NMR- and GC-MS-based metabolite profiling, illustrating that each individual was characterized by a specific metabolite profile differing in composition and time courses as well as levels of the metabolites produced. Some metabolites such as 3-hydroxybenzoic acid and 3,5-dihydroxybenzoic acid were only detected in incubations of samples from a few subjects. Other metabolites varied significantly in the levels produced and their appearance over time as shown by the coefficients of variations (CV) ranging between 39 and 265\% (average $\mathrm{CV}=0.91$ ) (Figure S3 of Supporting Information) and the individual kinetic curves for several metabolites provided in Figures 1-3 and in the Supporting Information (Figure S4 of Supporting Information), respectively. Principal component analysis (PCA) revealed no clustering of groups (data not shown) and no (except one) significant correlations of the AUC values of various metabolites between the subjects were found (Figure S3 of Supporting Information), showing that the metabolite profiles differ between subjects. A central example demonstrating the interindividual variability of polyphenol bioconversion is the production of 3-phenylpropionic acid and its hydroxylated derivatives (Figure 2). 3-Phenylpropionic acid is a typical end metabolite that was produced for most subjects except for subjects 6 and 9 who did not produce or even produced lower levels of 3-phenylpropionic acid as compared to blank conditions. Similarly, 3-(3hydroxyphenyl)propionic acid was detected as an end metabolite although in lower amounts. In contrast, 3-(4-hydroxyphenyl)propionic acid was only temporarily produced for most subjects except for subjects 6,7 , and 9, for which this metabolite was still present after $72 \mathrm{~h}$ of fermentation. The temporary runs of the kinetic curves from subject 3 suggest the conversion from 3-(4-hydroxyphenyl)propionic acid and 3-(3-hydroxyphenyl)propionic acid to 3-(phenyl)propionic acid. For other subjects, this conversion may be less efficient or even inhibited as for subjects 6 and 9, who did not produce 3-phenylpropionic acid.

Different Metabolites Are Formed from Black Tea or Red Wine/ Grape Polyphenols by Human Gut Microbiota. Clear differences 


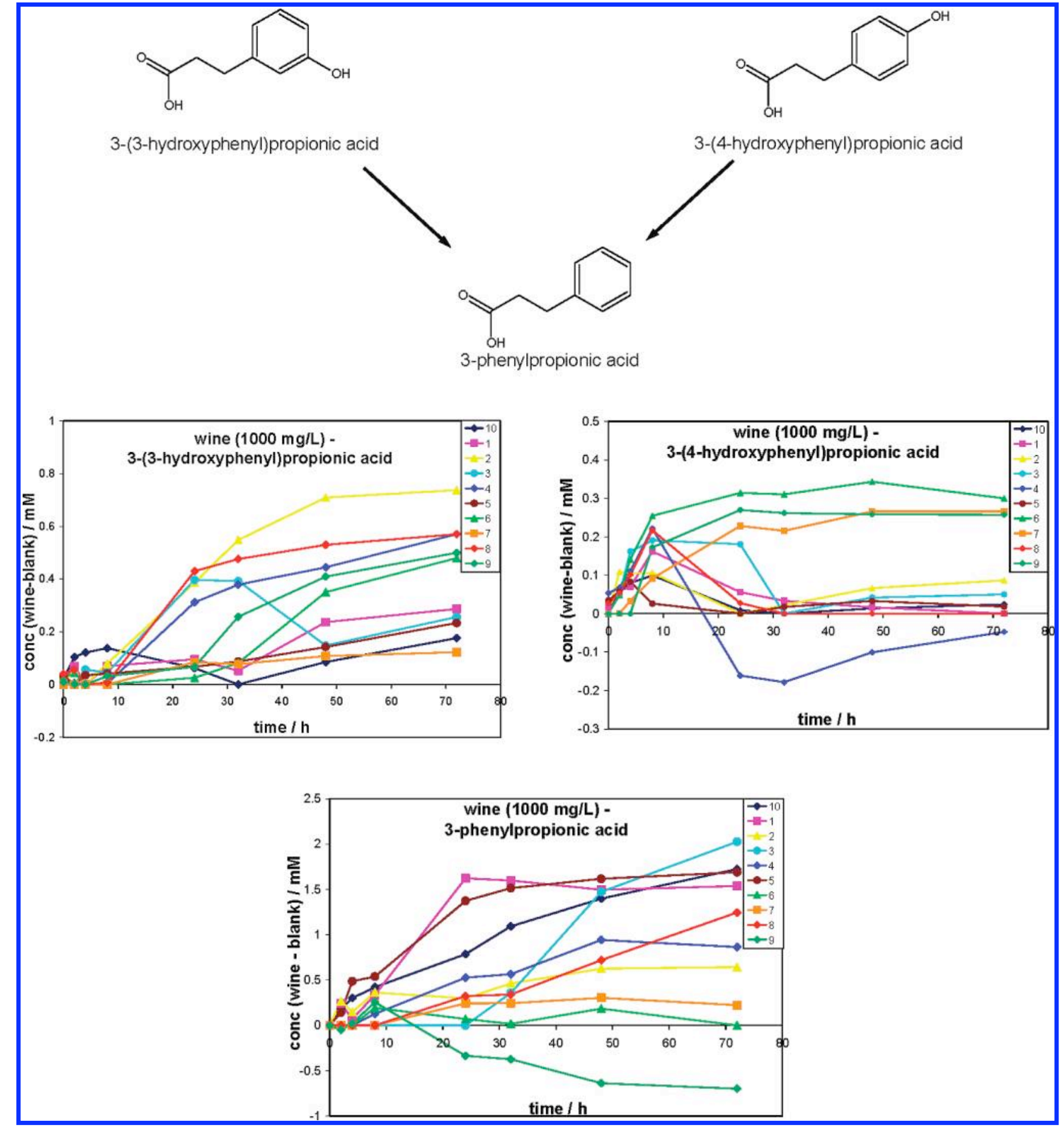

Figure 2. Bioconversion of 3-(3-hydroxyphenyl)propionic acid (left panel) and 3-(4-hydroxyphenyl)propionic acid (right panel) to 3-phenylpropionic acid (middle panel) from wine/grape extract as derived from ${ }^{1} \mathrm{H}$ NMR spectra and initial concentrations of $1000 \mathrm{mg} / \mathrm{L}$. The kinetic curves from each subject are displayed by different colors. The concentration derived from the blank experiment was subtracted from the concentration derived from the experiments using the wine/grape extract (conc (wine-blank)/mM).

were observed between the microbial metabolites that were produced from the black tea and red wine/grape polyphenols, respectively. For instance, the metabolites pyrogallol and gallic acid were exclusively found for the fermentation of black tea polyphenols (Figure 1), with gallic acid already being present in the initial tea extract. Furthermore, vanillic acid was hardly detected in samples incubated with black tea polyphenols, whereas this compound was present in small amounts in the initial red wine/grape extract and clearly produced as an intermediate metabolite from the wine/grape polyphenols (Figure 3). On the contrary, 2,6-dihydroxybenzoic acid was exclusively present after incubation with black tea extract (Table 1).
The differences between the conversion of black tea and red wine/grape polyphenols were not only reflected in the production of different metabolites but also in the produced levels. For example, on average, higher levels of 3-(3-hydroxyphenylpropionic acid) were produced from the wine/grape extract than from the tea extract, whereas the opposite was found for the production of 3-hydroxyphenylacetic acid (Figure 3).

The Complex Polyphenol Mixtures Are Converted to a Limited Number of Key Metabolites by Human Gut Microbiota. The polyphenol extracts used in the present study are complex mixtures the compositions of which were only known to a limited extent due to 

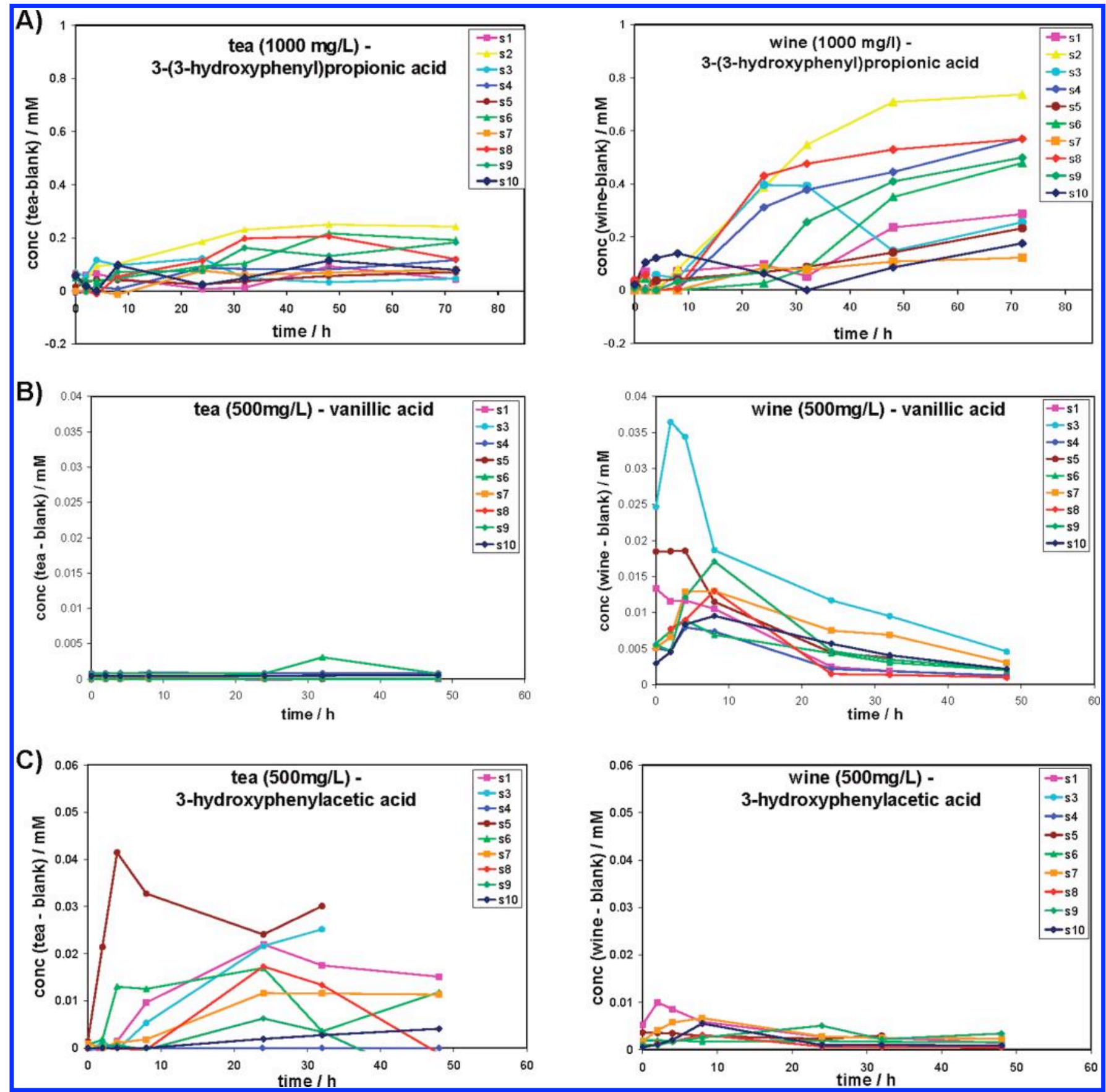

Figure 3. Differences in the bioconversion of black tea (left panels) and wine/grape polyphenols (right panels) as observed for 3-(3-hydroxyphenyl)propionic acid as determined by ${ }^{1} \mathrm{H} \mathrm{NMR}(\mathbf{A})$, vanillic acid (B), and 3-hydroxyphenylacetic acid (C), as determined by and GC-MS. The kinetic curves from each subject are displayed by different colors. The concentration derived from the blank experiment was subtracted from the concentration derived from the experiments using the wine and tea extracts, respectively (conc (wine-blank); (conc (wine-blank)/mM; conc (tea-blank)/mM).

analytical obstacles to identify oligomeric polyphenols. For example, only $20-30 \%$ of the compositions of the red wine and grape juice extracts compositions were identified by liquid chromatography using commercially available standards. The major fraction consisted of oligomeric proanthocyanidins. Similarly, the major fraction of flavonoids in the black tea extract included unidentified thearubigines. The phenolic components that were quantified in the tea and wine/grape extract are listed in Table S2 of Supporting Information.

These complex mixtures were converted to a variety, but still limited number of metabolites summarized in Table 1. The major metabolites produced were 3-phenylpropionic acid and its hydroxylated derivatives followed by phenylacetic acid and its hydroxylated derivatives. Benzoic acids were generally produced in lower amounts. This result indicates that diverse polyphenols were funnelled to a set of key metabolites potentially by using similar polyphenol bioconversion pathways.

Incubation with Polyphenols Has an Impact on Bacterial Production of SCFAs. Several SCFAs were quantified from NMRbased metabolite profiles (Figure 5). The levels of acetic acid were significantly increased upon fermentation with black tea polyphenols and to a lesser extent after incubation with the wine/grape extract. Butyric acid concentrations tended to increase after fermentation with both polyphenol extracts. The levels of propionic acid did not change during the fermentations with polyphenols. In contrast, isobutyric acid and isovaleric acid tended to decrease after fermentation with the tea extract. 


\section{DISCUSSION}

In the current study, batch culture fermentations on fecal samples from 10 healthy individuals were performed to assess the interindividual variability of polyphenol metabolism by gut microbiota and to compare the microbial bioconversion of red wine/grape and black tea polyphenols. Most importantly, substantial interindividual differences in the production of numerous bacterial polyphenol metabolites were observed. This interindividual variability was characterized by the production of different metabolites, by different levels of the intermediate and end products as well as by variable time courses, suggesting that each individual generates a specific microbial metabolome. This may be attributed to different bacterial populations present in the individual fecal microbial communities, harboring specific enzymatic capacities and therefore reflect microbial diversity among individuals (21). In agreement with this, each individual fecal sample was characterized by a distinct microbial composition $(R<0.32$, average $\mathrm{CV}=0.69)$ as determined by HITChip analysis, an oligonucleotide microarray for phylogentic profiling of human intestinal tract communities (22) (Tables S3, S4 of Supporting Information).

Besides the diversity of the gut microbes and their complex interplay, individual dietary habits may have caused slightly varying levels of initial polyphenol concentrations and thus slight differences in the metabolite production.

The interindividual variability in the production of metabolites might also be due to several cleavage possibilities. For example, quercetin or quercetin-3-rutinoside present in black tea (23) can be broken down to 3-hydroxyphenylacetic acid, 3-(3-hydroxyphenyl)propionic acid, and 3,4-dihydroxbenzoic acid $(17,24)$. In general, 3-(3-hydroxyphenyl)propionic acid was predominantly produced, whereas 3-hydroxybenzoic acid was only marginally present, indicating that $\beta$-oxidation was not a major bacterial degradation route. This is in line with earlier indications that $\beta$-oxidation of dietary flavonoids only occurs in host tissues (25). In vivo, 3-phenylpropionic acid is known to be absorbed by the host, usually converted to benzoic acid, conjugated with glycine to hippuric acid that is finally excreted in urine (26). In comparison, hydroxylated phenylacetic acids are not further metabolized after absorption or methylated in the liver before their urinary excretion (23).

Furthermore, different kinetics of the polyphenol degradation may contribute to the interindividual variability in the metabolite profiles. For instance, fast or inhibited conversion of 3-(4-hydroxyphenyl)propionic acid to 3-phenylpropionic acid (Figure 2) was observed, which is in line with previous studies indicating differences in the ability of the intestinal microbiota of individual rats and humans to dehydroxylate 3-(4-hydroxyphenyl)propionic acid to 3-phenylpropionic acid with a corresponding impact on the urinary excretion of downstream metabolites $(26,27)$. Also the conversion from 3-(3-hydroxyphenyl)propionic acid to 3-phenylpropionic acid, which has been described earlier in literature $(17,28)$, was more or less efficient potentially depending on the individual microbiota composition. Another example for different conversion rates is the degradation from gallic acid to pyrogallol, which is known to be absorbed by the host and further excreted as pyrogallol sulfate in urine in vivo (29). In the present in vitro study, however, pyrogallol was further degraded potentially via pholorglucinol to butyrate and acetate (30). Because the temporary production of pyrogallol was only observed at the high initial concentration of black tea extract, the degradation of gallic acid is most likely a fast process. Similarly, phloroglucinol was not detected in samples from all individuals (Figure S4 of Supporting Information) and hence may indicate a highly efficient conversion rather than a reduced production of this compound, as phloroglucinol is known to be rapidly degraded by intestinal bacterial species (31).

Interestingly, the wine/grape and tea extracts with their complex polyphenol compositions were fermented to a limited number of key metabolites, in particular to 3-phenylpropionic acid and its hydroxylated derivatives. This observation indicates a funnel process using common degradation pathways for diverse polyphenols and is in line with other findings on dietary fibers, suggesting that different bacterial colonic communities share general biochemical characteristics metabolizing different substrates to specific metabolic patterns (32). The funnel process is illustrated in Figure 4, displaying the total amounts of diverse metabolites produced per individual and extract as well as conversion steps from several polyphenols known to be present in the extracts. For example, various polyphenols such as caffeic acid and catechin are known to be degraded to 3-(3,4-dihydroxyphenyl)propionic acid which can be further dehydroxylated to 3-(3-hydroxyphenyl)propionic acid and finally to 3-phenylpropionic acid $(17,33,34)$. Another example is 3,4 dihydroxyphenylacetic acid, which is known to be a degradation product from quercetin and procyanidin (dimer) $(23,35)$. Furthermore, 3-(4-hydroxyphenyl)propionic acid can originate from $p$-coumaric acid and catechin, while 3,4-dihydroxybenzoic acid is a known metabolite from anthocyanidins, catechin, and quercetin $(17,36,37)$. The funnel process also became apparent in the total amount of metabolites produced within $72 \mathrm{~h}$. For example, in most cases higher amounts of 3-(4-hydroxyphenyl)propionic acid were generated than the initial amount of $p$-coumaric acid present in the tea extract, indicating that 3-(4-hydroxyphenyl)propionic acid was also produced from other precursors. This funnel process may enhance the interindividual variability in the metabolite production and hamper the prediction of metabolite profiles from complex polyphenol mixtures. On the other hand, structurally closely related compounds present both in the tea and wine/grape extracts may also partly explain why microbial bioconversion of the different polyphenol sources resulted in the formation of typical central end products. For instance, the basic building blocks of larger proanthocyanidins and thearubigins (polymerized theaflavins) are catechins, which are concatenated in a different manner and to a different degree (38).

Despite the interindividual differences, both polyphenol extracts were clearly distinguishable in their metabolite profiles, demonstrating that bioconversion also depended on the precursor substances. For example, the formation of pyrogallol and 2,6-dihydroxybenzoic acid could be primarily ascribed to polyphenols from black tea, whereas vanillic acid was only detected in profiles of samples incubated with the wine/grape extract. Moreover, the amounts of specific metabolites such as 3-(3-hydroxyphenyl)propionic acid varied between the two polyphenol extracts. This variation can be attributed to the different composition of the two polyphenol extracts and is in agreement with previous reports on various microbial metabolites produced from tea or wine polyphenols such as proanthocyanidins, catechins, and tea flavonoids (33). Considering that the conversion of diverse polyphenols after ingestion generates a limited number of key metabolites that potentially exert health benefits on the host rather than the initial compounds, future studies are recommended to focus on exploring the downstream effects of these metabolites on the host.

In addition to the formation of phenolics, the incubation with especially black tea extract resulted in significantly elevated levels of acetate and a slight increase in the production of butyrate (Figure 5). The latter may be of interest due to beneficial health effects that have been associated with butyrate production in the colon (39). 


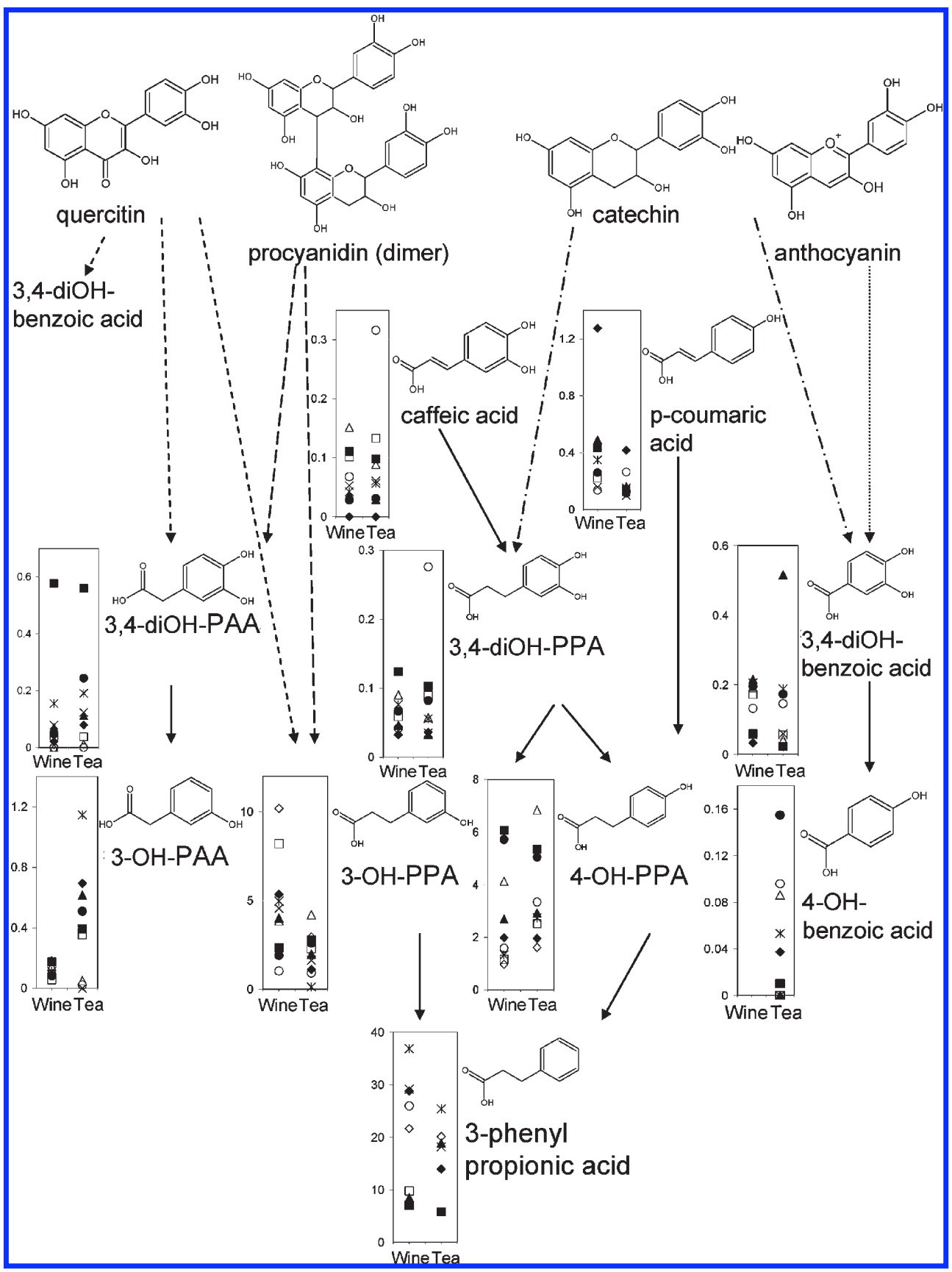

Figure 4. Illustration of the funnel process indicating the bioconversion of several polyphenols present in the tea and/or wine/grape extracts, such as quercetin, procyanidin (dimer), catechin, anthocyanin, caffeic acid, and p-coumaric acid to a variety of phenolic acids detected by ${ }^{1} \mathrm{H}$ NMR and GC-MS. The arrows indicate cleavage possibilities of different polyphenols. In the smaller diagrams, the total amounts of metabolites (AUC in $\mathrm{mM} \cdot \mathrm{h}$ ) produced within $48 \mathrm{~h}$ of fermentation of $500 \mathrm{mg} / \mathrm{L}$ polyphenol extract are plotted for the tea and wine/grape extracts, respectively, and for each individual as indicated by different symbols. (4-OH-benzoic acid: 4-hydroxybenzoic acid; 3,4-diOH-benzoic acid: 3,4-dihydroxybenzoic acid; 3-OH-PAA: phenylacetic acid; 3,4-diOH-PAA: 3,4-dihydroxyphenylacetic acid; 3-OH-PPA: 3-(3-hydroxyphenyl)propionic acid, 4-OH-PPA: 3-(4-hydroxyphenyl)propionic acid; 3,4-diOH-PPA: 3-(3,4dihydroxyphenyl)propionic acid).

Furthermore, the levels of branched-chain fatty acids such as isovalerate and isobutyrate tended to be reduced after fermentation of the black tea extract (Figure 5). The decreased production of isobutyrate is in line with earlier in vivo results from human volunteers who received the same wine/grape extract (14). The effects on the production of SCFAs may be caused by the degradation of the polyphenols. For example, butyrate has been identified earlier to be produced from quercetin and other flavonoids by microorganisms isolated from human feces $(18,24)$ and from catechin and tannic acid after batch fermentations with rat cecal inoculum (40).
Furthermore, sugar moieties typically conjugated to polyphenols in plant material (the polyphenol extracts were not pre-digested before the fermentations) and other non-phenolic substances present in the tea and wine/grape extracts are likely to have contributed to the observed effects on SCFAs.

In this study, the combination of NMR- and GC-MS-based profiling technologies provided a strong methodological advantage, allowing the detection of a wide range of polyphenol metabolites in order to gain comprehensive insight into the complex colonic metabolism of polyphenols. Our data suggest that the 


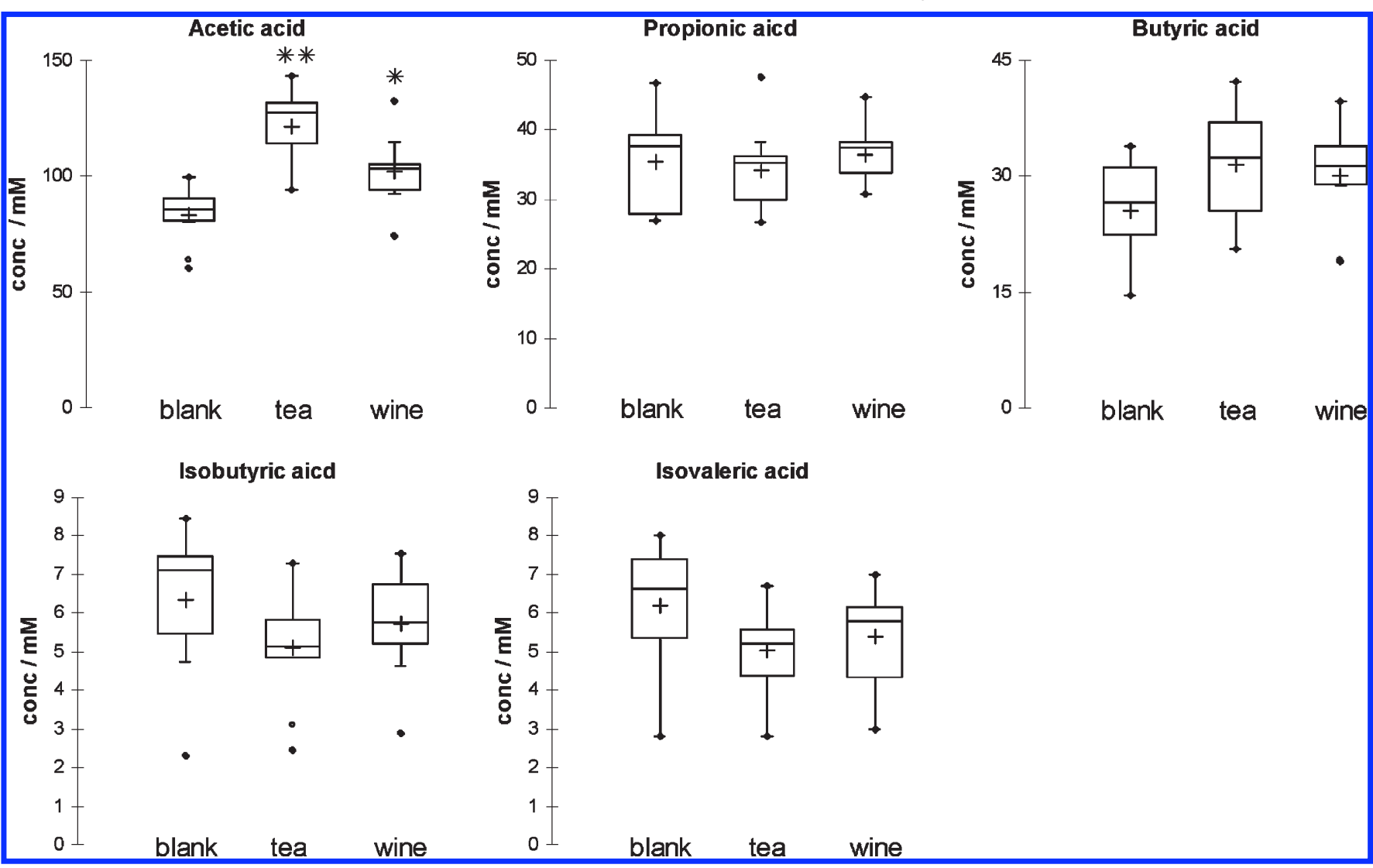

Figure 5. Box plots depicting the concentrations of SCFAs (derived from ${ }^{1} \mathrm{H}$ NMR spectra) after $72 \mathrm{~h}$ of fecal batch fermentation without polyphenols added (indicated with blank) and with black tea (tea) and wine/grape (wine) polyphenols at a concentration of $1000 \mathrm{mg} / \mathrm{L}$, respectively. Statistically significant differences in the results of the polyphenol treatments compared to blank conditions are indicated with asterisks ( ${ }^{\star *} P<0.01$; ${ }^{*} P<0.05$ ).

diversity of polyphenols present in the wine/grape and tea extract were funnelled through common degradation routes to a limited number of metabolites that may be beneficial to the host. Each individual generated a specific metabolite pattern, implying that potential health effects may also vary among subjects. These metabolite patterns may be related to specific microbial compositions. The identification of microbes involved in polyphenol metabolism is challenging considering that several microbial species may be involved in the production of one metabolite due to metabolic cross-feeding mechanisms and/or common enzymatic properties shared by different microbial species. So far, only some intestinal bacterial groups with polyphenol-metabolizing capacities have already been identified, e.g. Eubacterium ramulus and Clostridium orbiscindens $(24,41)$. Our study design was not sufficiently powered to associate specific polyphenol metabolites with microbial groups present in the initial fecal samples by taking into account multivariate factors. Future studies on larger cohorts may allow for exploring the link between the microbial diversity and polyphenol metabolism. In this respect, functiondriven metagenomics seems to be a powerful approach to identify functional anchors in complex microbial communities and may increase the power due to the potential functional redundancy of activities in the gut community $(42,43)$. Furthermore, in vitro batch culture fermentations represent artificial conditions with unknown validity in vivo. Future studies employing subjectmatched data analysis will be required to validate our findings in vivo and to establish coherent links between in vitro bioconversion and in vivo bioavailability of polyphenols.

In conclusion, this study has unraveled individual microbial bioconversion processes of complex polyphenol mixtures from black tea and red wine/grape and outlines the extent of interindividual variability and differences due to polyphenol sources. Importantly, the current findings demonstrate that metabolite profiles vary among individuals, which may imply that corresponding health benefits depending on microbial bioconversion of dietary polyphenols cannot be generalized. Furthermore, the results highlight key degradation products and potentially interesting bioactive compounds. Ultimately, this study is a first step toward a better understanding of how dietary polyphenols may be involved in beneficial health effects for the human body.

\section{ACKNOWLEDGMENT}

We thank Dr. Robèr Kemperman for providing the data of the HITChip analysis.

Supporting Information Available: Phenolic acid standards used for GC-MS analysis; quantified phenolic components in black tea and wine/grap extracts determined by LC-MS analysis; microbial composition of fecal samples from 10 individuals; signal intensities of HITChip probes detecting divers microbial species or groups for each of the 10 individuals; correlation matrix among the subjects calculated from univariance-scaled, relative concentrations of the microbial species from HITChip analysis; bioconversion of black tea polyphenols $(1000 \mathrm{mg} / \mathrm{L})$ by the microbiota of subject 1; production of 3-(4-hydroxyphenyl)propionic acid from black tea and wine/grape polyphenols as determined by NMR spectroscopy; representation of inter-individual variation in metabolite production from the fermentation with black tea and wine/ grape extract; kinetic curves of diverse phenolic metabolites from black tea and wine/grape polyphenols derived from GC-MS profiles. This material is available free of charge via the Internet at http://pubs.acs.org. 


\section{LITERATURE CITED}

(1) Halliwell, B.; Rafter, J.; Jenner, A. Health promotion by flavonoids, tocopherols, tocotrienols, and other phenols: direct or indirect effects? Antioxidant or not? Am. J. Clin. Nutr. 2005, 81, 268S-276S.

(2) Manach, C.; Williamson, G.; Morand, C.; Scalbert, A.; Remesy, C. Bioavailability and bioefficacy of polyphenols in humans. I. Review of 97 bioavailability studies. Am. J. Clin. Nutr. 2005, 81, 230S-242S.

(3) Selma, M. V.; Espin, J. C.; Tomas-Barberan, F. A. Interaction between phenolics and gut microbiota: role in human health. J. Agric. Food Chem. 2009, 57, 6485-6501.

(4) Possemiers, S.; Grootaert, C.; Vermeiren, J.; Gross, G.; Marzorati, M.; Verstraete, W.; van de Wiele, T. The intestinal environment in health and disease-recent insights on the potential of intestinal bacteria to influence human health. Curr. Pharm. Des. 2009, 15, 2051-2065.

(5) Rajilic-Stojanovic, M.; Smidt, H.; de Vos, W. M. Diversity of the human gastrointestinal tract microbiota revisited. Environ. Microbiol. 2007, 9, 2125-2136.

(6) Tappenden, K. A.; Deutsch, A. S. The physiological relevance of the intestinal microbiota - contributions to human health. J. Am. Coll. Nutr. 2007, 26, 679S-683S.

(7) Forester, S. C.; Waterhouse, A. L. Metabolites are key to understanding health effects of wine polyphenolics. J. Nutr. 2009, 139 , 1824S-1831S.

(8) Possemiers, S.; Bolca, S.; Eeckhaut, E.; Depypere, H.; Verstraete, W. Metabolism of isoflavones, lignans and prenylflavonoids by intestinal bacteria: producer phenotyping and relation with intestinal community. FEMS Microbiol. Ecol. 2007, 61, 372-383.

(9) Luczaj, W.; Skrzydlewska, E. Antioxidative properties of black tea. Prev. Med. 2005, 40, 910-918.

(10) Gonthier, M. P.; Cheynier, V.; Donovan, J. L.; Manach, C.; Morand, C.; Mila, I.; Lapierre, C.; Remesy, C.; Scalbert, A. Microbial aromatic acid metabolites formed in the gut account for a major fraction of the polyphenols excreted in urine of rats fed red wine polyphenols. J. Nutr. 2003, 133, 461-467.

(11) van de Wiele, T.; Boon, N.; Possemiers, S.; Jacobs, H.; Verstraete, W. Inulin-type fructans of longer degree of polymerization exert more pronounced in vitro prebiotic effects. J. Appl. Microbiol. 2007, 102, $452-460$.

(12) Macfarlane, G. T.; Macfarlane, S. Models for intestinal fermentation: association between food components, delivery systems, bioavailability and functional interactions in the gut. Curr. Opin. Biotechnol. 2007, 18, 156-162.

(13) Jacobs, D. M.; Gaudier, E.; van Duynhoven, J.; Vaughan, E. E. Non-digestible food ingredients, colonic microbiota and the impact on gut health and immunity: a role for metabolomics. Curr. Drug Metab. 2009, 10, 41-54.

(14) Jacobs, D. M.; Deltimple, N.; van Velzen, E.; van Dorsten, F. A.; Bingham, M.; Vaughan, E. E.; van Duynhoven, J. (1)H NMR metabolite profiling of feces as a tool to assess the impact of nutrition on the human microbiome. NMR Biomed. 2008, 21, 615-626.

(15) Grun, C. H.; van Dorsten, F. A.; Jacobs, D. M.; Le, B. M.; van Velzen, E. J.; Bingham, M. O.; Janssen, H. G.; Van Duynhoven, J. P. GC-MS methods for metabolic profiling of microbial fermentation products of dietary polyphenols in human and in vitro intervention studies. J. Chromatogr. B: Analvt. Technol. Biomed. Life Sci. 2008, $871,212-219$.

(16) Bolca, S.; Possemiers, S.; Herregat, A.; Huybrechts, I.; Heyerick, A.; De Vriese, S.; Verbruggen, M.; Depypere, H.; De Keukeleire, D.; Bracke, M.; De Henauw, S.; Verstraete, W.; van de Wiele, T. Microbial and dietary factors are associated with the equol producer phenotype in healthy postmenopausal women. J. Nutr. 2007, 137, 2242-2246.

(17) Rechner, A. R.; Smith, M. A.; Kuhnle, G.; Gibson, G. R.; Debnam, E. S.; Srai, S. K.; Moore, K. P.; Rice-Evans, C. A. Colonic metabolism of dietary polyphenols: influence of structure on microbial fermentation products. Free Radical Biol. Med. 2004, 36, 212-225.

(18) Schneider, H.; Schwiertz, A.; Collins, M. D.; Blaut, M. Anaerobic transformation of quercetin-3-glucoside by bacteria from the human intestinal tract. Arch. Microbiol. 1999, 171, 81-91.
(19) Scalbert, A.; Manach, C.; Morand, C.; Remesy, C.; Jimenez, L. Dietary polyphenols and the prevention of diseases. Crit. Rev. Food Sci. Nutr. 2005, 45, 287-306.

(20) Kohri, T.; Matsumoto, N.; Yamakawa, M.; Suzuki, M.; Nanjo, F.; Hara, Y.; Oku, N. Metabolic fate of (-)-[4-(3)H]epigallocatechin gallate in rats after oral administration. J. Agric. Food Chem. 2001, 49, 4102-4112.

(21) Zoetendal, E. G.; Rajilic-Stojanovic, M.; de Vos, W. M. Highthroughput diversity and functionality analysis of the gastrointestinal tract microbiota. Gut 2008, 57, 1605-1615.

(22) Rajilic-Stojanovic, M.; Heilig, H. G.; Molenaar, D.; Kajander, K.; Surakka, A.; Smidt, H.; de Vos, W. M. Development and application of the human intestinal tract chip, a phylogenetic microarray: analysis of universally conserved phylotypes in the abundant microbiota of young and elderly adults. Environ. Microbiol. 2009, 11, 1736-1751.

(23) Olthof, M. R.; Hollman, P. C.; Buijsman, M. N.; van Amelsvoort, J. M.; Katan, M. B. Chlorogenic acid, quercetin-3-rutinoside and black tea phenols are extensively metabolized in humans. $\underline{\text { J. Nutr. }}$. 2003, 133, 1806-1814.

(24) Schoefer, L.; Mohan, R.; Schwiertz, A.; Braune, A.; Blaut, M. Anaerobic degradation of flavonoids by Clostridium orbiscindens. Appl. Environ. Microbiol. 2003, 69, 5849-5854.

(25) Hollman, P. C.; Katan, M. B. Absorption, metabolism, and bioavailability of flavonoids. Flavonoids in Health and Disease; RiceEvans, C. A., Packer, L., Eds.; Marcel Dekker, Inc.: New York, Basel, 1998; pp 483-522.

(26) Clifford, M. N.; Copeland, E. L.; Bloxsidge, J. P.; Mitchell, L. A. Hippuric acid as a major excretion product associated with black tea consumption. Xenobiotica: Fate Foreign Compd. Biol. Svst. 2000, 30 , 317-326.

(27) Williams, R. E.; Eyton-Jones, H. W.; Farnworth, M. J.; Gallagher, R.; Provan, W. M. Effect of intestinal microflora on the urinary metabolic profile of rats: a (1)H-nuclear magnetic resonance spectroscopy study. Xenobiotica: Fate Foreign Compd Biol. Svst. 2002, 32, 783-794.

(28) Chesson, A.; Provan, G. J.; Russell, W. R.; Scobbie, L.; Richardson, A. J.; Stewart, C. Hydroxycinnamic acids in the digestive tract of livestock and humans. J. Sci. Food Agric. 1999, 79, 373-378.

(29) Daykin, C. A.; Van Duynhoven, J. P.; Groenewegen, A.; Dachtler, M.; van Amelsvoort, J. M.; Mulder, T. P. Nuclear magnetic resonance spectroscopic based studies of the metabolism of black tea polyphenols in humans. J. Agric. Food Chem. 2005, 53, 14281434.

(30) Krumholz, L. R.; Crawford, R. L.; Hemling, M. E.; Bryant, M. P. Metabolism of gallate and phloroglucinol in Eubacterium oxidoreducens via 3-hydroxy-5-oxohexanoate. J. Bacteriol. 1987, 169, 1886-1890.

(31) Braune, A.; Engst, W.; Blaut, M. Degradation of neohesperidin dihydrochalcone by human intestinal bacteria. J. Agric. Food Chem. 2005, 53, 1782-1790.

(32) Mortensen, P. B.; Clausen, M. R.; Bonnen, H.; Hove, H.; Holtug, K. Colonic fermentation of ispaghula, wheat bran, glucose, and albumin to short-chain fatty acids and ammonia evaluated in vitro in 50 subjects. JPEN J. Parenter. Enteral Nutr. 1992, 16, 433-439.

(33) Aura, A. M.; Mattila, I.; Seppänen-Laakso, T.; Miettinen, J.; Oksman-Caldentey, K. M.; Oresic, M. Microbial metabolism of catechin stereoisomers by human faecal microbiota: comparison of targeted analysis and a non-targeted metabolomics method. Phvtochem. Lett. 2008, 1, 18-22.

(34) Deprez, S.; Brezillon, C.; Rabot, S.; Philippe, C.; Mila, I.; Lapierre, C.; Scalbert, A. Polymeric proanthocyanidins are catabolized by human colonic microflora into low-molecular-weight phenolic acids. J. Nutr. 2000, 130, 2733-2738.

(35) Appeldoorn, M. M.; Vincken, J. P.; Aura, A. M.; Hollman, P. C.; Gruppen, H. Procyanidin dimers are metabolized by human microbiota with 2-(3,4-dihydroxyphenyl)acetic acid and 5-(3,4-dihydroxyphenyl)-gamma-valerolactone as the major metabolites. J. Agric. Food Chem. 2009, 57, 1084-1092.

(36) Aura, A. M. Microbial metabolism of dietary phenolic compounds in the colon. Phvtochem. Rev. 2008, 7, 407-429. 
(37) Gonthier, M. P.; Donovan, J. L.; Texier, O.; Felgines, C.; Remesy, C.; Scalbert, A. Metabolism of dietary procyanidins in rats. Free Radical Biol. Med. 2003, 35, 837-844.

(38) Santos-Buelga, C.; Scalbert, A. Proanthocyanidins and tannin-like compounds - nature, occurrence, dietary intake and effects on nutrition and health. J. Sci. Food Agric. 2000, 80, 1094-1117.

(39) Wong, J. M.; de, S. R.; Kendall, C. W.; Emam, A.; Jenkins, D. J. Colonic health: fermentation and short chain fatty acids. J. Clin. Gastroenterol. 2006, 40, 235-243.

(40) Bravo, L.; Abia, R.; Eastwood, M. A.; Saura-Calixto, F. Degradation of polyphenols (catechin and tannic acid) in the rat intestinal tract. Effect on colonic fermentation and faecal output. Br. J. Nutr. 1994, 71, 933-946.

(41) Schneider, H.; Blaut, M. Anaerobic degradation of flavonoids by Eubacterium ramulus. Arch. Microbiol. 2000, 173, 71-75.

(42) Tap, J.; Mondot, S.; Levenez, F.; Pelletier, E.; Caron, C.; Furet, J. P.; Ugarte, E.; Munoz-Tamayo, R.; Paslier, D. L.; Nalin, R.; Dore, J.; Leclerc, M. Towards the human intestinal microbiota phylogenetic core. Environ. Microbiol. 2009, 11, 2574-2584.
(43) van Duynhoven, J.; Vaughan, E. E.; Jacobs, D. M.; Kemperman, R.; van Velzen, E. J.; Gross, G.; Roger, L. C.; Possemiers, S.; Smilde, A. K.; Dore, J.; Westerhuis, J. A.; van de Wiele, T. Microbes and Health Sackler Colloquium: Metabolic fate of polyphenols in the human superorganism. Proc. Natl. Acad. Sci. U.S.A. 2010, DOI: 10.1073/pnas. 1000098107.

Received for review April 19, 2010. Revised manuscript received July 22, 2010. Accepted August 3, 2010. We acknowledge the financial support of the European Community under the Marie Curie Transfer of Knowledge-Industry-Academia Strategic Partnership Scheme, MTKICT-2006-042786 (GUTSYSTEM), especially for the secondment of Dr. Gabriele Gross to Gent University. Part of this project was carried out within the research program of The Netherlands Metabolomics Centre (NMC) which is part of The Netherlands Genomics Initiative/Netherlands Organization for Scientific Research. The authors have declared no conflict of interest. 